\title{
Evaluación fisicoquímica, microbiológica y sensorial de una salsa y una bebida funcional a base de extracto de frijol rojo (phaseolus vulgaris) con pulpa de guayaba en el municipio de Valledupar
}

\author{
Physico-chemical, microbiological and sensory evaluation of a sauce and a \\ functional drink based on red bean extract (phaseolus vulgaris) with pulp of \\ guayaba in the municipality of Valledupar
}

\author{
Eswin J. Flórez ${ }^{1}$, Jenny C. Vinchira², Zuray S. Acosta ${ }^{3}$.
}

\section{Palabras Claves:}

Frijol, pulpa, guayaba, néctar, bebida, funcional, extracto.

\section{Resumen}

Objetivo: evaluar de manera fisicoquímica, microbiológica y sensorial una salsa y una bebida funcional a base de extracto de fríjol rojo (phaseolus vulgaris) con pulpa de guayaba.

Metodología: el trabajo se desarrolló en una planta piloto, donde se tuvieron en cuenta la pulpa de la fruta y granos de frijoles rojos comercializados en los diferentes supermercados de la ciudad de Valledupar donde se tomaron muestras representativas de 5 kilos de guayaba y 5 kilos de fríjol para la obtención de la bebida y, con base en la NTC 659, se aplicó un diseño factorial de 22.

Resultados: La consistencia de la salsa de extracto de fríjol rojo con mayor contenido de proteína $(11,3 \%)$ fue el atributo de mayor aceptación por parte de los evaluadores no entrenados con 67,3 \%, y el color fue el de menor aceptación con $53,3 \%$. Sin embargo, en forma general, el producto tuvo una preferencia de $61 \%$. El sabor de la bebida tipo néctar con mayor contenido de proteína $(11 \%)$ a base de extracto de fríjol rojo y extracto de guayaba fue el atributo que tuvo el mayor porcentaje de aceptación por parte de los evaluadores no entrenados, con $70,6 \%$, y la consistencia fue el atributo con menor porcentaje de aceptación con 61,3. Sin embargo, otra vez en forma general, considerados todos los atributos, la bebida tuvo una preferencia de $64,5 \%$.

Conclusiones: la composición mineral ( $\mathrm{Fe}, \mathrm{Na}, \mathrm{K}$, Ca y Mg) tanto de la salsa de extracto de fríjol como de la bebida a base de extracto de frijol rojo y extracto de guayaba fueron aportes valiosos, sobre todo en el contenido del potasio y del calcio, que contribuyen a mantener la estabilidad muscular y gástrica del ser humano.

\section{Abstract}

Objective: to evaluate in a physicochemical, microbiological and sensory way a sauce and a functional drink based on red bean extract (phaseolus vulgaris) with guava pulp.

Methodology: the work was developed in a pilot plant, which took into account the pulp of fruit and beans of red beans sold in the different supermarkets of the city of Valledupar where representative samples of 5 kilos of guava and 5 kilos of beans to obtain the drink and, based on NTC 659, a factorial design of 22 was applied.

\section{Keywords:}

Beans, pulp, guava, nectar, drink, functional, extract.
Results: The consistency of the red bean extract sauce with the highest protein content (11.3\%) was the attribute of greatest acceptance by the untrained evaluators with $67.3 \%$, and the color was the one with the lowest acceptance with $53.3 \%$. However, in general, the product had a preference of $61 \%$. The flavor of the nectar-like drink with the highest protein content $(11 \%)$ based on red bean extract and guava extract was the attribute that had the highest percentage of acceptance by the untrained evaluators, with $70.6 \%$, and consistency was the attribute with the lowest percentage of acceptance with 61.3. However, again in a general way, considering all the attributes, the drink had a preference of $64.5 \%$.

Conclusions: the mineral composition ( $\mathrm{Fe}, \mathrm{Na}, \mathrm{K}, \mathrm{Ca}$ and $\mathrm{Mg}$ ) both of the bean extract sauce and the drink based on red bean extract and guava extract were valuable contributions, especially in the content of potassium and calcium, which contribute to maintaining the muscular and gastric stability of the human being.

1Universidad Popular del Cesar, correo electronico: eflorezavendano@yahoo.com

2 Universidad Popular del Cesar. Correo electronico:jenny.vinchira@co.nestle.com

3 Autor de correspondencia, Universidad Popular del Cesar, Correo electronico: zhurayacosta@hotmail.com recibido: 22 de marzo de 2017, aceptado: 27 de junio de 2017

Como citar este artículo: E. Flórez, J. Vinchira., y Z. Costa, "Evaluacion fisicoquimica, microbiologica y sensorial de una salsa y una bebida funcional a base de extracto de frijol rojo (Phaseolus vulgaris) con pulpa de Guayaba en el municipio de Valledupar", Investigación e Innovación en Ingenierias, vol. 6, n. 1, pp. 70 - 83., 2018.DOI: https://10.17081/invinno.6.1.2776 


\section{Introducción}

El fríjol es una de las leguminosas más importantes en el mundo [1]. En Colombia tiene gran impacto por su volumen de producción, valor alimenticio, generación de empleo y amplia aceptación en la dieta popular con 3,86 kg de consumo por persona al año. En 2001, el fríjol generó un ingreso bruto aproximado para los agricultores colombianos de US\$ 98`922.935, además de 12,5 millones de jornales directos, en los que participaron cerca de 125.000 productores. Sin embargo, a pesar de ser un país con variabilidad genética en fríjol, con cultura de producción y adaptación del cultivo a variados ambientes, presenta una reducción y déficit en la oferta para suplir el mercado interno, razón por la cual el país se ve obligado a realizar importaciones para cubrir la demanda nacional [2].

El fríjol común es una planta anual, herbácea, intensamente cultivada desde la zona tropical hasta las templadas. Es originario de América y se le conoce con diferentes nombres: poroto, haricot, caraota, judía, aluvia, habichuela y otros [3]. El frijol, por ejemplo, es uno de los alimentos básicos en la dieta del costarricense y es la principal fuente de proteína; es rico en lisina, pero deficiente en los aminoácidos azufrados: metionina, cistina y triptófano; por lo cual una dieta adecuada en aminoácidos esenciales se logra al combinar fríjol con cereales (arroz, maíz, otros) [4].

Por otra parte, la baja incidencia de enfermedad cardiovascular en Francia llevó al desarrollo de estudios sobre este fenómeno, demostrándose que la fruta de mayor consumo tiene niveles altos de flavonoides, elementos polifenoles derivados de los difenilpiranos vegetales [5]. Este compuesto puede contribuir al efecto ateroprotector, como lo sugieren diferentes trabajos que muestran asociación entre su ingesta y bajos niveles poblacionales de enfermedad vascular periférica y cardiovascular [6]. Como ocurre en ese país europeo, en las islas del Caribe, Centroamérica y la región norte de América del Sur se presenta un alto nivel de consumo de fríjol rojo (Phaseolus vulgaris), alimento con un alto porcentaje de flavonoides, que puede ser el responsable de las bajas incidencias de enfermedades cardiovasculares en esta parte del planeta $[6,7,8]$. 


\section{Materiales y metodos}

Este trabajo de investigación se desarrolló en una planta piloto, el Centro de Investigación y Desarrollo de la Ingeniería de la Universidad Popular del Cesar, con los siguientes objetivos:

- Cuantificar algunas características fisicoquímicas del extracto de fríjol rojo y pulpa de guayaba.

- Formular una salsa y bebida con las proporciones de pulpa de guayaba y el extracto de fríjol rojo.

- Determinar algunos parámetros fisicoquímicos de los productos (salsa y bebida).

- Observar el comportamiento microbiológico de la salsa y la bebida.

- Analizar sensorialmente la salsa y la bebida elaborada.

Para esta investigación se tuvieron en cuenta la pulpa de la fruta y granos de frijoles rojos comercializados en los diferentes supermercados de la ciudad de Valledupar, de donde se tomaron muestras representativas de alrededor de 5 kilos de guayaba y 5 kilos de fríjol para la obtención de la bebida y, con base en la NTC 659, se aplicó un diseño factorial de 22, donde los factores fueron: A: $\%$ de pulpa de guayaba y B: \% de extracto de fríjol rojo, con los niveles 10, 12 y 10, 6, respectivamente y tres repeticiones también respectivas, con posterior análisis microbiológico de coniformes totales, mesófilos, clostridium, hongos y levaduras. En la elaboración de la salsa con base en la norma técnica colombiana 921, se aplicó un diseño completamente al azar con tres tratamientos y tres repeticiones, donde la variable de salida fue el porcentaje de proteína, todo ello con un análisis de medias por Tukey y un nivel de confianza de $95 \%$. Además, tanto para la bebida como para la salsa, se seleccionó estadísticamente el tratamiento de mayor aporte proteico.

\section{Caracterización fisicoquímica del extracto del fríjol, salsa y bebida}

\begin{tabular}{|l|l|l|}
\hline VARIABLES & TÉCNICAS & MÉTODOS \\
\hline $\mathrm{pH}$ & Potenciometría & $10,041 / 84$ de la AOAC., \\
\hline Brix & Refractometría & 22,024 de la AOAC \\
\hline Ceniza & Gravimetría & $942.05 / 90$ de la AOAC \\
\hline Humedad & Gravimetría & $930.15 / 90$ de la AOAC \\
\hline Proteína bruta & Kjeldahl & $955.04 / 90$ de la AOAC \\
\hline Fibra & Digestión Acida & $962.09 / 90$ de la AOAC \\
\hline
\end{tabular}

Tabla 1.

Parámetros y métodos de la caracterización fisicoquímica del extracto del fríjol, salsa y bebida 
Los tratamientos de la salsa y la bebida que tuvieron mejores resultados estadísticos desde el punto de vista proteico, se sometieron a una prueba de aceptación de 5 puntos (me gusta, me gusta mucho, me es indiferente, me disgusta y me disgusta mucho) para el aroma, sabor, consistencia y color.

La prueba se aplicó a 30 evaluadores no entrenados de ambos sexos y edades comprendidas entre 18 y 45 años, tomados al azar, usándose como instrumento una encuesta para obtener los datos necesarios para el análisis estadístico.

\section{Resultados y discusión}

\section{Resultados de la pulpa de frijol y pulpa de guayaba}

Análisis fisicoquímicos de la pulpa de fríjol

En la Figura 1 se muestra el comportamiento de algunos parámetros fisicoquímicos del extracto de fríjol rojo

Figura 1

Comportamiento de los parámetros fisicoquímico de la pulpa de fríjol rojo

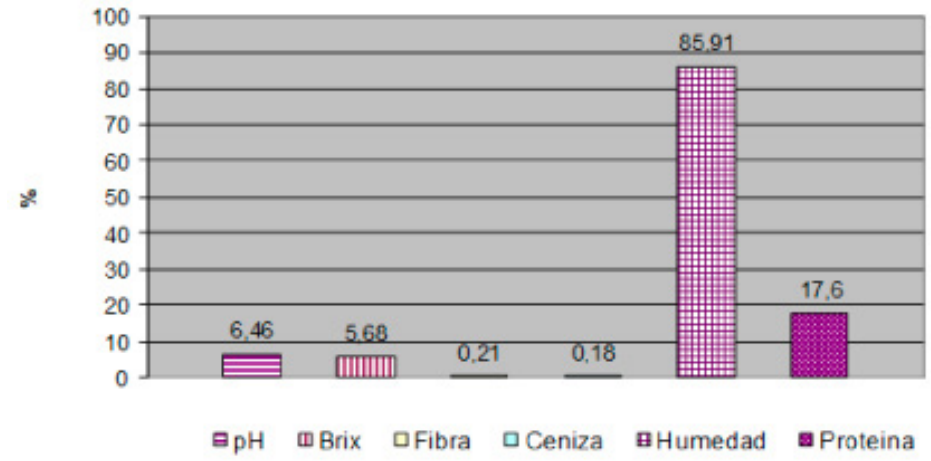

Dicha figura revela todos los componentes nutricionales estudiados en el extracto del frijol, y en concreto, a la proteína con 17,6 \% como el nutriente de mayor concentración y a la fibra con 0,21 $\%$ como el de menor contenido. El valor de la proteína es de alrededor de $2 \%$ por debajo del valor reportado en la teoría y el de la fibra de un $95 \%$. Es posible que esto haya ocurrido porque no se aprovechó todo el frijol, sino que se utilizó solamente el extracto, quedando en el colado y exprimido un gran porcentaje de nutrientes.

\section{Análisis fisicoquímicos de pulpa de guayaba}

En la Figura 2 se muestra el resultado de algunos parámetros fisicoquímicos de la pulpa de guayaba utilizada en la estandarización de la bebida. 


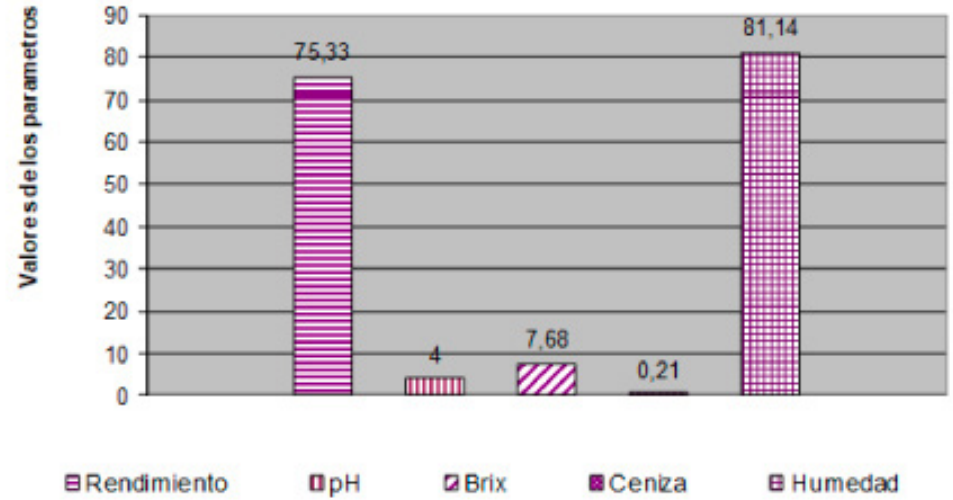

Figura 2

Comportamiento de los parámetros fisicoquímicos de la pulpa de guayaba

Se observa que, en promedio, la guayaba tiene el 75,33\% de rendimiento, 4 de $\mathrm{pH}, 7,68$ de ${ }^{\circ}$ Brix y $81,14 \%$ de humedad. Los minerales se encuentran dentro del rango expuesto en la teoría.

\section{Resultados de la formulación de los productos salsa y bebida}

Salsa de extracto de frijol rojo

Con base en la norma técnica colombiana 921, se estandarizó o se formuló la salsa a partir de la pulpa o extracto del frijol rojo:

\begin{tabular}{|l|l|l|l|l|l|l|l|l|l|}
\hline T & $\begin{array}{l}\% \\
\text { Pulpa }\end{array}$ & $\begin{array}{l}\% \\
\text { Azúcar }\end{array}$ & $\begin{array}{l}\% \\
\text { CMC }\end{array}$ & $\begin{array}{l}\% \\
\text { sal }\end{array}$ & $\begin{array}{l}\% \\
\text { Ácido } \\
\text { cítrico }\end{array}$ & $\begin{array}{l}\% \\
\text { Agua }\end{array}$ & $\begin{array}{l}\% \\
\text { Benzoato }\end{array}$ & $\begin{array}{l}\% \\
\text { Sorbato }\end{array}$ & $\begin{array}{l}\% \\
\text { Ácido } \\
\text { acético }\end{array}$ \\
\hline T1 & 70 & 10 & 0,07 & 2 & 0,03 & 49,10 & 0,03 & 0,03 & 14 \\
\hline T2 & 80 & 10 & 0,07 & 2 & 0,03 & 47,21 & 0,03 & 0,03 & 14 \\
\hline T3 & 90 & 10 & 0,07 & 2 & 0,03 & 52,31 & 0,03 & 0,03 & 14 \\
\hline
\end{tabular}

\section{Tabla 2}

Formulación de la salsa de extracto de frijol Fuente: Los investigadores.

\begin{tabular}{|l|l|l|l|l|}
\hline Tratamientos & $\begin{array}{l}\% \\
\text { Canela }\end{array}$ & $\begin{array}{l}\% \\
\text { Tomillo }\end{array}$ & $\begin{array}{l}\% \\
\text { Laurel }\end{array}$ & $\begin{array}{l}\% \\
\text { Clavo }\end{array}$ \\
\hline T1 & 0,1 & 0,2 & 0,2 & 0,1 \\
\hline T2 & 0,1 & 0,2 & 0,2 & 0,1 \\
\hline T3 & 0,1 & 0,2 & 0,2 & 0,1 \\
\hline
\end{tabular}

Tabla 3

Formulación de las especias de salsa de extracto de fríjol Fuente: Los investigadores. 


\section{Bebida (tipo néctar)}

Para la estandarización de este producto se hizo uso de la norma técnica colombiana 659, de donde se obtuvo:

\begin{tabular}{|l|l|l|l|l|l|l|l|l|}
\hline Tratamientos & $\begin{array}{l}\% \\
\text { Pulpa }\end{array}$ & $\begin{array}{l}\% \\
\text { Azúcar }\end{array}$ & $\begin{array}{l}\% \\
\text { CMC }\end{array}$ & $\begin{array}{l}\% \\
\text { Ácido } \\
\text { Ascórbico }\end{array}$ & $\begin{array}{l}\% \\
\text { Água }\end{array}$ & $\begin{array}{l}\% \\
\text { Benzoato }\end{array}$ & $\begin{array}{l}\% \\
\text { Sorbato }\end{array}$ & $\begin{array}{l}\% \\
\text { pectina }\end{array}$ \\
\hline a1b1 & 20 & 13,60 & 0,07 & 0,03 & 66,18 & 0,03 & 0,03 & 0,03 \\
\hline a1b2 & 16 & 10,88 & 0,07 & 0,03 & 72,22 & 0,03 & 0,03 & 0,03 \\
\hline a2b1 & 22 & 14,96 & 0,07 & 0,03 & 63,53 & 0,03 & 0,03 & 0,03 \\
\hline a2b2 & 18 & 12.24 & 0,07 & 0,03 & 68,06 & 0,03 & 0,03 & 0,03 \\
\hline
\end{tabular}

Tabla 4

Formulación de la bebida de extracto de frijol y pulpa de guayaba

Fuente: Autores.

\section{Resultados fisicoquímicos de la salsa de fríjol rojo}

En la Tabla 5, se encuentran los resultados de proteína, fibra, ceniza y humedad en las diferentes formulaciones o tratamientos de la salsa.

Tabla 5

Proteína,

fibra, ceniza y

humedad en las

formulaciones o tratamientos de la

salsa

\begin{tabular}{|l|l|l|l|l|}
\hline Variables & T1 & T2 & T3 & Media \\
\hline a1b1 & $11,03 a$ & $10,94 b$ & $10,98 b$ & 10,98 \\
\hline a1b2 & $1,53 b$ & $1,59 a$ & $1,61 a$ & 1,57 \\
\hline a2b1 & $2,36 b$ & $2,29 b$ & $2,30 b$ & 2,31 \\
\hline a2b2 & $49,86 a$ & $49,97 a$ & $49,90 a$ & 49,90 \\
\hline
\end{tabular}

T1 : formulación 1 de la salsa de fríjol rojo

$\mathrm{T} 2$ : formulación 2 de la salsa de fríjol rojo

T3 : formulación 3 de la salsa de fríjol rojo

Valores con letras diferentes en la misma fila difieren

significativamente

El análisis de varianza reveló diferencias significativas para la proteína, fibra y ceniza, dependiendo del tratamiento. El tratamiento T1 presentó en este sentido el mayor valor de proteína con 11,03 $\%$, diferenciándose estadísticamente de los tratamientos T2 y T3, los cuales arrojaron los menores porcentajes de proteína. Estos porcentajes de proteína se hallan por debajo de los presentados por la pulpa de fríjol, lo que posiblemente se deba a que solo se aprovechó el extracto y no toda la pulpa, 
donde la proteína no se solubilizó lo suficiente, pasando poca en el filtrado o colado. El tratamiento T3 presentó el mayor valor de Fibra con \% 1, 61, diferenciándose estadísticamente de los tratamientos T1 y T2, que arrojaron los menores porcentajes de fibra. Esto se dio porque el mayor porcentaje de extracto de fríjol (90\%) se le asignó al tratamiento número tres, y a mayor concentración de pulpa aumenta la presencia de este nutriente. Por su parte, ell tratamiento T1 presentó el mayor valor de ceniza con \% 2, 36, diferenciándose estadísticamente de los tratamientos T2 y T3. Es posible que esto haya sucedido así por efecto de la temperatura, que pudo favorecer la disponibilidad de los minerales en el tratamiento número 1, formulado con $70 \%$ de extracto de fríjol rojo, que en el resto de las formulaciones o tratamientos. Según el análisis de la varianza del \% de humedad de los tratamientos, no se presentaron diferencias significativas, lo que sugiere que si se selecciona cualquiera de los tres tratamientos de la salsa de extracto de frijol rojo, el porcentaje de humedad, será estadísticamente el mismo.

Composición mineral de la salsa con mayor aporte proteico

La Figura 3 refleja que el potasio con $900 \mathrm{mg} / 100 \mathrm{~g}$ de muestra seca es el mineral con mayor presencia en la salsa de extracto de fríjol, mientras que el de menor presencia fue el sodio, con $12 \mathrm{mg}$ / $100 \mathrm{~g}$, resultados similares a los reportados en el fundamento teórico de este trabajo.

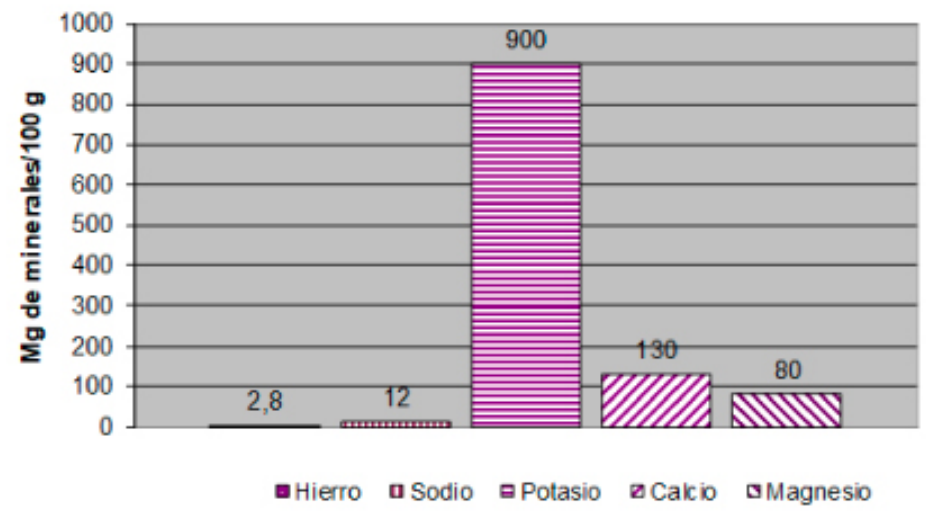

Figura 3

Comportamiento mineral de la salsa con mayor contenido proteico

\section{Resultados del análisis sensorial (prueba de aceptación) de la salsa de fríjol}

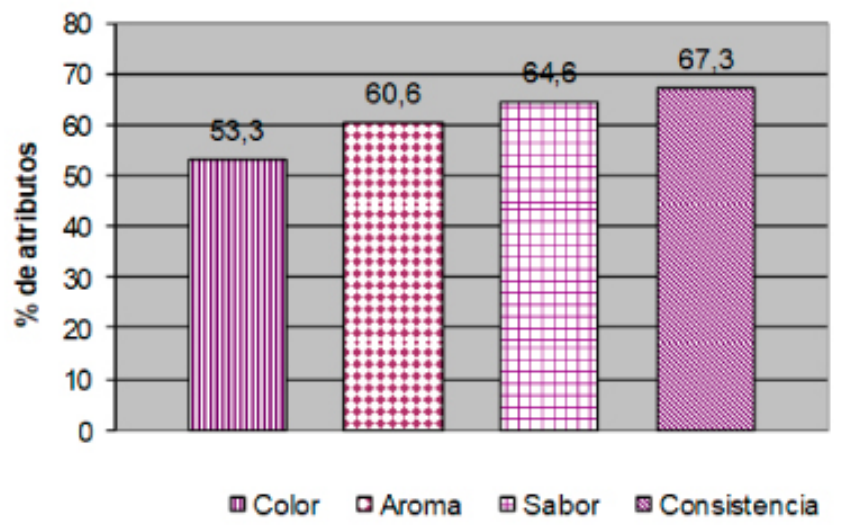

\author{
Figura 4 \\ Comportamiento \\ sensorial de la \\ salsa con mayor \\ aporte proteico
}


La figura correspondiente al análisis sensorial arrojó que el atributo con menor aceptación fue el aroma y el de mayor aceptación, la consistencia; sin embargo, en forma general , se puede afirmar que la salsa con mayor porcentaje de proteína tuvo por parte de los evaluadores una preferencia del $61 \%$.

\section{Comportamiento de algunos parámetros fisicoquímico de la salsa con mayor aporte proteico}

Tabla 6

Comportamiento del $\mathrm{pH}$, acidez y

los grados brix de

la salsa con mayor aporte proteico

\begin{tabular}{|c|c|c|c|c|c|c|c|c|}
\hline \multicolumn{3}{|c|}{1 día } & \multicolumn{3}{|c|}{15 días } & \multicolumn{3}{|c|}{30 días } \\
\hline $\mathrm{pH}$ & Brix & $\begin{array}{l}\text { Acidez } \\
\text { titulable }\end{array}$ & $\mathrm{pH}$ & Brix & $\begin{array}{l}\text { Acidez } \\
\text { titulable }\end{array}$ & $\mathrm{pH}$ & Brix & $\begin{array}{l}\text { Acidez } \\
\text { titulable }\end{array}$ \\
\hline 2,7 & 26 & 0,27 & 2,6 & 26 & 0,28 & 2,5 & 26 & 0,29 \\
\hline
\end{tabular}

En el Cuadro 5 se observa que los parámetros fisicoquímicos $\mathrm{pH},{ }^{\circ}$ brix y acidez al día 1, así como a los 15 y 30 días no representan cambios significativos, ya que están en el rango establecido por la norma técnica colombiana 921, lo que garantiza la vida útil del producto.

\section{Resultados microbiológicos de la salsa con mayor aporte proteico}

En estos resultados se observa que las colonias de microorganismos analizados en la salsa en el día 1, así como a los 15 y 30 días no representan riesgos para la salud humana, ya que están por debajo de las UFC exigidas por la norma técnica colombiana 659, por lo que el producto resulta apto para el consumo humano en los 30 días.

\section{Determinación de la vida útil de la salsa}

La salsa elaborada presentó una vida útil de 30 días bajo condiciones de almacenamiento a una temperatura de entre 4 y $7^{\circ} \mathrm{C}$. Los parámetros de acidez titulable, $\mathrm{pH}$ y sólidos solubles no cambiaron significativamente durante el estudio. En cuanto al color, olor, sabor, apariencia y consistencia, la salsa proteica presentó buena aceptación. Los resultados microbiológicos se encuentran entonces dentro de los rangos aceptados para la salsa con una duración máxima de 30 días, según lo establecido por la Norma Técnica Colombiana NTC 921.

\section{Resultados de la bebida a base de fríjol rojo y pulpa de guayaba}

Resultados fisicoquímicos de la bebida a hase de frínl rnio 1/ mulna do ouarıaha

\section{Figura 5}

Comportamiento de la proteína, fibra, ceniza y humedad de la bebida en las cuatro tratamientos combinaciones o

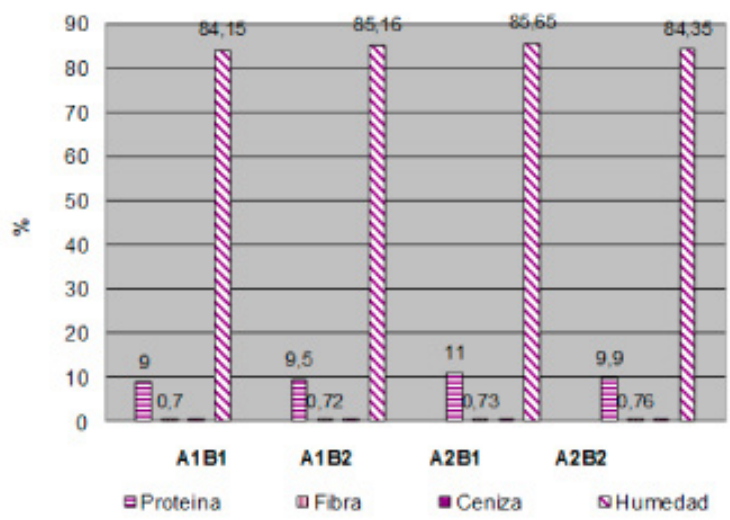

Como citar este artículo: E. Flórez, J. Vinchira., y Z. Costa, "Evaluacion fisicoquimica, microbiologica y sensorial de una salsa y una bebida funcional a base de extracto de frijol rojo (Phaseolus vulgaris) con pulpa de Guayaba en el municipio de Valledupar", Investigación e Innovación en Ingenierias, vol. 6, nº. 1, pp. 70 - 83., 2018.DOI: https://10.17081/invinno.6.1.2776 
El análisis de varianza de la bebida reveló diferencias significativas para la proteína, fibra y ceniza, dependiendo del tratamiento o las combinaciones. La combinación A2B1 presentó el mayor valor de proteína con $11 \%$, diferenciándose estadísticamente de las combinaciones o tratamientos A1B1, A1B2 y A2B2, las cuales arrojaron los menores porcentajes de proteína. Esto se presentó porque el mayor porcentaje de extracto de fríjol estuvo en el tratamiento A2B1.

La combinación A2B2 presentó el mayor valor de fibra con 0,76 \%, diferenciándose estadísticamente de A1B1, A1B2 y A2B1, que arrojaron los menores porcentajes de fibra. Es posible que esto se deba al aporte de fibra de la pulpa de guayaba, ya que el tratamiento o combinación A2B2 tuvo uno de los mayores porcentajes de esta pulpa. También pudo deberse a que, al momento del colado y exprimido del extracto del frijol, la transferencia de este nutriente fue mayor.

La combinación A1B2 presentó el mayor valor de ceniza con $0,23 \%$, diferenciándose estadísticamente de A1B1, A2B2 y A2B1, que arrojaron los menores porcentajes de ceniza. Ello ocurrió porque hubo mayor solubilidad de los minerales al momento del licuado del fríjol y de la guayaba, lo que produjo en el colado, y por lo tanto en el mezclado para obtener la combinación A1B2, mayor transferencia de minerales.

Según el análisis de la varianza de la bebida, el porcentaje de humedad de las combinaciones no presentó diferencias significativas.

Composición mineral de la bebida con mayor aporte proteico

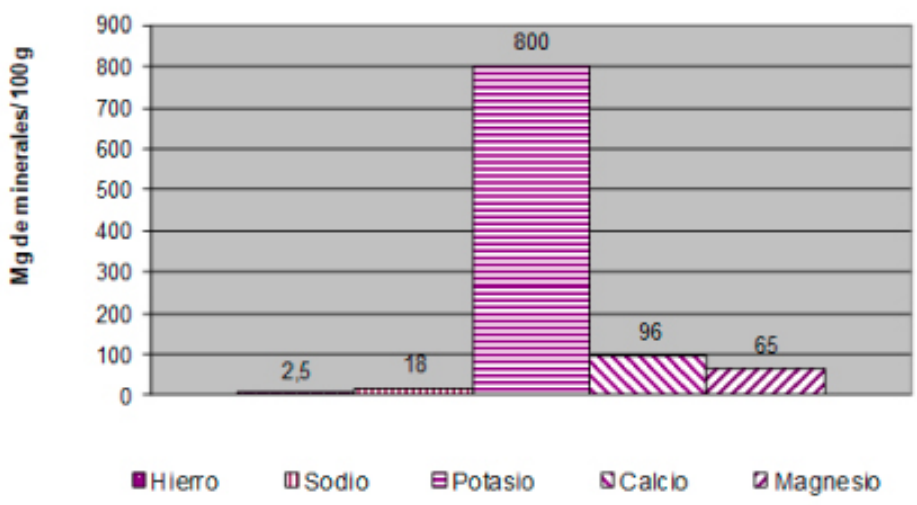

Figura 4

Comportamiento sensorial de la salsa con mayor aporte proteico

El análisis estadístico reflejó que el potasio, con 800 mg / 100 g de muestra seca, es el mineral de mayor presencia en la bebida de extracto de fríjol y extracto de guayaba, así como el de menor presencia es el sodio con $18 \mathrm{mg} / 100 \mathrm{~g}$, resultados similares a los reportados por Alubia y colcol. en el fundamento teórico.

\section{Determinación de la vida útil de la bebida}

La bebida elaborada presentó una vida útil de 30 días bajo condiciones de almacenamiento a una temperatura entre 4 y $7^{\circ} \mathrm{C}$. Los parámetros de acidez titulable, $\mathrm{pH}$ y sólidos solubles no cambiaron significativamente durante el estudio. En cuanto al color, olor, sabor, apariencia y consistencia, la 
bebida proteica presentó buena aceptación. Los resultados microbiológicos se encuentran dentro de los rangos aceptados para la bebida tipo néctar de frutas con una duración máxima de 30 días, según lo establecido por la Norma Técnica Colombiana NTC 659.

Comportamiento del $\mathrm{pH}$, acidez y los grados brix en la bebida con mayor aporte proteico

El siguiente análisis (Cuadro 6) está basado en el monitoreo y/o seguimiento de la bebida tipo néctar durante su respectivo almacenamiento. El cuadro muestra los cambios en cuanto a ${ }^{\circ}$ Brix, acidez y $\mathrm{pH}$ en los diferentes periodos de tiempo, es decir, 1 día, 15 días y 30 días después de estar elaboradas y almacenadas a temperatura entre 4 y $7^{\circ} \mathrm{C}$ aproximadamente

Tabla 7

Comportamiento

del $\mathrm{pH}$, acidez y

los grados brix de

la salsa con mayor aporte proteico

\begin{tabular}{|l|l|l|l|l|l|l|l|l|}
\hline \multicolumn{4}{|l|}{1 día } & \multicolumn{2}{l|}{15 días } & \multicolumn{2}{l|}{30 días } \\
\hline pH & Brix & $\begin{array}{l}\text { Acidez } \\
\text { titulable }\end{array}$ & $\mathrm{pH}$ & Brix & $\begin{array}{l}\text { Acidez } \\
\text { titulable }\end{array}$ & $\mathrm{pH}$ & Brix & $\begin{array}{l}\text { Acidez } \\
\text { titulable }\end{array}$ \\
\hline 2,9 & 11 & 0,26 & 2,7 & 11 & 0,27 & 2,5 & 11 & 0,29 \\
\hline
\end{tabular}

En él se observa que los parámetros fisicoquímicos $\mathrm{pH}$, acidez y 0Brix de la bebida, al día1, a los 15 días y a los 30 días, no representan cambios significativos, ya que están en el rango establecidos por la norma técnica colombiana 659. Lo anterior garantiza la vida útil del producto.

Resultados microbiológicos de la bebida con mayor aporte proteico

En estos resultados se observa que las colonias de microorganismos analizados en la bebida de fríjol rojo y pulpa de guayaba, en los días 1, 1530 día no representan riesgos para la salud humana, ya que se hallan por debajo de las UFC de la norma técnica colombiana 659, por lo que la vida útil del producto se puede considerar en 30 días.

\section{Resultados de la prueba sensorial o de aceptación de la bebida}

El análisis sensorial arrojó que el atributo con menor aceptación fue el aroma con 61,3 \% y el de mayor aceptación fue el sabor, con 70,6 \%; sin embargo, en forma general, la bebida tipo néctar con mayor porcentaje de proteína obtuvo una preferencia del $64,5 \%$.

\section{Figura 7}

Comportamiento sensorial de la bebida tipo néctar con mayor aporte proteico

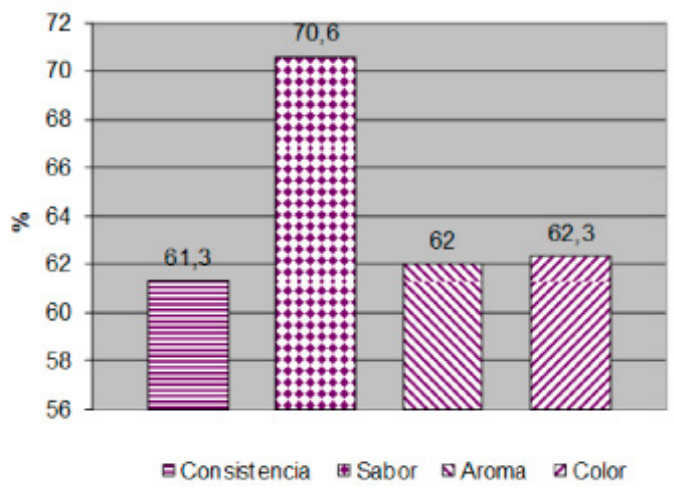

Como citar este artículo: E. Flórez, J. Vinchira., y Z. Costa, “Evaluacion fisicoquimica, microbiologica y sensorial de una salsa y una bebida funcional a base de extracto de frijol rojo (Phaseolus vulgaris) con pulpa de Guayaba en el municipio de Valledupar", Investigación e Innovación en Ingenierias, vol. 6, nº. 1, pp. 70 - 83., 2018.DOl: https://10.17081/invinno.6.1.2776 
Los parámetros fisicoquímicos de la formulación de la bebida tipo néctar que resultó con mayor aporte proteico, al comparar los dos productos comerciales no presentaron diferencias significativas en cuanto al parámetro acidez, pero sí en cuanto a los parámetros ${ }^{\circ} \mathrm{Brix}$ y $\mathrm{pH}$, ya que la bebida presentó un $\mathrm{pH}$ y ${ }^{\circ}$ Brix menor en comparación con los néctares comerciales (véase cuadro 7). A pesar de esto, la bebida se encuentra dentro de los parámetros fisicoquímicos adoptados por la Norma Técnica Colombiana NTC 659.

\begin{tabular}{|l|l|l|l|l|}
\hline NÉCTARES & $\mathrm{pH}$ & 0Brix & $\%$ Acidez & $\%$ Proteína \\
\hline $\begin{array}{l}\text { Néctar de } \\
\text { uvito brasilero }\end{array}$ & 3,50 & 15,22 & 0,38 & 0,70 \\
\hline $\begin{array}{l}\text { Néctar } \\
\text { comercial } \\
\text { (éxito) }\end{array}$ & 3,45 & 10,20 & 0,20 & 1,00 \\
\hline $\begin{array}{l}\text { Bebida tipo } \\
\text { néctar (fríjol y } \\
\text { guayaba) }\end{array}$ & 2,70 & 11,00 & 0,27 & 9,85 \\
\hline
\end{tabular}

Tabla 8

Comparación del $\mathrm{pH}$, OBrix, acidez y proteína de la bebida más proteica tipo néctar con otras bebidas o néctares Fuente: Los investigadores. 


\section{Conclusión}

El extracto de fríjol rojo reveló en promedio 6,46 de $\mathrm{pH}$, unos ${ }^{\circ}$ Brix de 5,68, 0,21\% de ceniza, un 0,18\% de fibra, $85,91 \%$ de humedad y $17,6 \%$ de proteína. La guayaba, por su parte, arrojó en promedio un rendimiento en pulpa del 75.33 por ciento, un $\mathrm{pH}$ de 4, unos sólidos solubles de 7,68, un porcentaje de ceniza de 0,21 y $81,14 \%$ de humedad.

El tratamiento 1 (70\% extracto de fríjol) de las formulaciones de la salsa de fríjol rojo fue el que mayor contenido de proteína presentó y, con base en los resultados fisicoquímicos y microbiológicos, tuvo una vida útil de 30 días. El T3 con 90 \% de extracto de fríjol rojo, fue el que presentó el mayor porcentaje de fibra.

De las cuatro formulaciones o tratamientos, la combinación A2B1 de la bebida con 12\% de extracto de fríjol y 10 \% de pulpa de guayaba fue la que arrojó mayor contenido de proteína, y al ser sometida a los análisis fisicoquímicos y microbiológicos, indicó una vida útil de 30 días con los parámetros fínales de la NTC. De las cuatro combinaciones de la bebida tipo néctar, la formulación A2B2 fue la que arrojó el mayor contenido de fibra [10-13].

El análisis sensorial indicó un 61 \% y 64,5 \% de aceptabilidad por parte de los evaluadores de la salsa de fríjol rojo y de la bebida tipo néctar, entre todos sus atributos, respectivamente.

La composición mineral de la salsa y de la bebida constituyeron aportes valiosos, sobre todo en el contenido del potasio (900 y $800 \mathrm{mg} / 100 \mathrm{~g}$ de muestra seca) y del calcio (130 y $96 \mathrm{mg} / 100 \mathrm{~g}$ de muestra seca), respectivamente, valores que contribuyen a mantener la estabilidad muscular y gástrica del ser humano.

Por último, el costo de producción de $250 \mathrm{ml}$ de salsa de fríjol fue de $\$ 1,743.21$ y el de $500 \mathrm{ml}$ de bebida tipo néctar a base de extracto de fríjol rojo y pulpa de guayaba, de \$3,637.287. Este resultó ser mucho mayor que un litro de néctar comercial, pero ello se justifica por la procedencia del contenido proteico que posee. 


\section{Referencias}

1. W. J. Broughton, G. Hernández, M. Blair S. Beebe, P. Gepts and J. Vanderleyden., "Beans (Phaseolus spp.) model food legumes", Plant and Soil, vol. 252, 2003.

2. Ministerio de comercio exterior. Productos agropecuarios. Colombia, 2003.

3. A.Barón, Articulo El cultivo del frijol, publicado por Slide player, Costarica, 2014.

4. Anuario estadístico agrícola, pecuario, acuícola, forestal y minero. Secretaría de Desarrollo Agropecuario y Minero en AGROEXPO - 2001, Bogotá.

5. S. Parra, MC. Fernández, T. Vandale y L. López, "Dietary fiber and gastro-intestinal cancer, implication for the mexican population”, Arch Latinoam Nutr, vol. 44, pp. 76-81., 1994.

6. T. Kanazawa, T. Osanai, XS. Zhang, et al. "Protective effects of soy protein on the peroxidizability of lipoproteins in cerebral vascular diseases”, J Nutr, vol. 125: pp.639S-646S., 199

7. H. Ulloa, J. Ulloa, H. Alam, Efectos antioxidante y antiagregante del fríjol rojo: un probable factor ateroprotector en la población latinoamericana., 2003.

8. Instituto Colombiano de Normas técnicas y certificación. NTC 659. Papel: formatos Bogotá, INCONTEC 1996 p.2.

9. Instituto Colombiano de Normas técnicas y certificación. NTC 921. Papel: formatos Bogotá, INCONTEC 1996 p.2.

10. Gavilán, Ramírez y Cabrera Efecto de la Fécula de Maíz en las características físico - químicas y organolépticas en la elaboración de Salsas Agridulces de Uva, 2005.

11. G. Mazza, Alimentos funcionales. Aspectos bioquímicos y de procesado. Acriba. Zaragoza. 2000.

12. M. Méndez Ramírez, C. Flores López, M. Reyes Vega, O. Rebolloso Padilla, M. Hernández González, X. Ruelas Chacón, Análisis sensorial descriptivo de salsas picantes tradicionales de la ciudad de Saltillo, Coahuila, 1990.

13. Y. Reyes cruz, Seminario ii alimentos funcionales en la nutrición humana: estrés oxidativa y fisiología gastrointestinal, 2004.

Bibliografía de consulta

A.O.A.C Association of Official Analytical Chemists. (AOAC). 1984. Official Methods of Analysis, 13th. Ed. Washington, D.C. 1990.

R. Alonso, L.A. Rubio, M. Muzquiz y F. Marzo, "Evaluación de la Disponibilidad de Minerales en Harinas de Frijol y en Mezclas de Maíz/Frijol Extrudidas”, Sci. Tech, vol. 94, pp. 1-13.,2001.

M. Ashwell, Concepts of Functional Foods. ILSI EUROPE CONCISE MONOGRAPH SERIES. Belgium. 2015.

R. Bellisle, AT. Diplock y G. Hornstra, "Functional Food science in Europe", Br J Nutr. No.80, pp. S3193. 2002.

Como citar este artículo: E. Flórez, J. Vinchira., y Z. Costa, “Evaluacion fisicoquimica, microbiologica y sensorial de una salsa y una bebida funcional a base de extracto de frijol rojo (Phaseolus vulgaris) con pulpa de Guayaba en el municipio de Valledupar", Investigación e Innovación en Ingenierias, vol. 6, n. 1, pp. 70 - 83., 2018.DOl: https://10.17081/invinno.6.1.2776 
A. Calvelo, Alimentos Funcionales Su posicionamiento y rotulación. La Alimentación Latinoamericana. 244:8-13. 2002.

MS. Anthony, TB. Clarkson, CL Jr. Hughes, TM. Morgan, GL. Burke, "Soy isoflavones improve cardiovascular risk factors without affecting the reproductive system of peripubertal rhesus monkeys", J Nutr, vol. 126, pp. 43-50, 1996

J. Jeffrey, "Savory fruit - based pances", Food Technology, vol. 50, n.1, pp. 70-73., 1996.

S. Kudou, Y. Fleury y D. Welti, "Malonyl Isoflavone glycosides in soybean seeds", Agric. Biol. Chem, vol.55, pp. 2227-2233, 1991.

Ministerio de Agricultura y Ganadería, Técnicos sobre Cuarenta y Cinco Cultivos Agrícolas de Costa Rica. Dirección General de Investigación y Extensión Agrícola. San José, Costa Rica., 1991.

C. Mora Nora, estudio de aglutininas en variedades phaseolus vulgaris, revista colombiana de ciencias químico farmacéutica, 2014.

J. J. Pérez Acero, Cultivos I (Cereales-Leguminosas-Oleaginosas). Facultad De Ciencias Agrarias De La Unad. Bogota D.C, 2000

S. Potter, "Overview of proposed mechanism for the hypocholesterolemic effect of soy", J Nutr, vol. 125, pp. 606S-611S, 1996.

M.C. Quicazán Cuenca. Aspectos Especiales De Las Condiciones Colombianas Para La Selección De Fuentes Alternas De Proteína. Profesora Asociada, Instituto De Ciencia Y Tecnología De AlimentosICTA. Universidad Nacional De Colombia. Sede Bogotá, 2015.

Resolución 7992 de 1991. Ministerio de salud. cap. iv néctares de frutas.

M. Roberfroid, "Concepts and strategy of functional food science: the European perspective", Am. J Clin Nutr, nº. 71, pp. 1660S-1664S, 2000.

J.A. Ross y C.M. Kasum, "Dietary flavonoids: bioavailability, metabolic effects, and safety", Annual Rewiews in nutrition, vol. 22, pp.19-32, 2002

A. Rozo, Ministerio de la Protección Social y el Instituto Nacional de Salud. Epidemióloga de la Dirección de Salud Pública del Ministerio, 2006.

F. Rolón, «Opciones de la banca comercial en productos de importación,» Dictamen Libre, n 12/13, pp. 71-75, 2013.

E. Sloan, "The top ten functional food trends", food technology, vol. 54, nº 4, pp. 33-62, 2000

L.A. Veríssimo Correa. Leguminosas Grano. Enciclopedia Practica De La Agricultura Y La Ganadería. Océano/Centrum, 2013.

Como citar este artículo: E. Flórez, J. Vinchira., y Z. Costa, “Evaluacion fisicoquimica, microbiologica y sensorial de una salsa y una bebida funcional a base de extracto de frijol rojo (Phaseolus vulgaris) con pulpa de Guayaba en el municipio de Valledupar", Investigación e Innovación en Ingenierias, vol. 6, nº. 1, pp. 70 - 83., 2018.DOl: https://10.17081/invinno.6.1.2776 